This item was submitted to Loughborough's Research Repository by the author.

Items in Figshare are protected by copyright, with all rights reserved, unless otherwise indicated.

\title{
Internet competency predicts practical hearing aid knowledge and skills in first-time hearing aid users
}

PLEASE CITE THE PUBLISHED VERSION

https://doi.org/10.1044/2016_AJA-16-0022

\section{PUBLISHER}

(C) American Speech-Language-Hearing Association

\section{VERSION}

AM (Accepted Manuscript)

\section{PUBLISHER STATEMENT}

This work is made available according to the conditions of the Creative Commons Attribution-NonCommercialNoDerivatives 4.0 International (CC BY-NC-ND 4.0) licence. Full details of this licence are available at: https://creativecommons.org/licenses/by-nc-nd/4.0/

\section{LICENCE}

CC BY-NC-ND 4.0

\section{REPOSITORY RECORD}

Maidment, David, William Brassington, Heather Wharrad, and Melanie Ferguson. 2019. "Internet Competency Predicts Practical Hearing Aid Knowledge and Skills in First-time Hearing Aid Users". figshare. https://hdl.handle.net/2134/36563. 


\section{Internet competency predicts practical hearing aid knowledge and skills in first-time hearing aid users.}

\begin{tabular}{|r|l|}
\hline Journal: & The American Journal of Audiology \\
\hline Manuscript ID & AJA-16-0022.R1 \\
\hline Manuscript Type: & Research Note \\
\hline Complete List of Authors: & $\begin{array}{l}\text { Maidment, David; National Institute of Health Research, Nottingham } \\
\text { Hearing Biomedical Research Unit } \\
\text { Brassington, William; Nottingham University Hospitals NHS Trust, } \\
\text { Nottingham Audiology Service } \\
\text { Wharrad, Heather; University of Nottingham, School of Health Sciences } \\
\text { Ferguson, Melanie; National Institute of Health Research, Nottingham } \\
\text { Hearing Biomedical Research Unit }\end{array}$ \\
\hline Keywords: & Hearing loss, Amplification or hearing aids, Outcomes \\
\hline Note: The following files were submitted by the author for peer review, but cannot be converted to \\
\hline You must view these files (e.g. movies) online.
\end{tabular}


Internet competency in hearing aid users

Internet competency predicts practical hearing aid knowledge and skills in first-time hearing aid users.

David Maidment $^{1}$, William Brassington ${ }^{2}$, Heather Wharrad ${ }^{3}$, Melanie Ferguson ${ }^{1,2}$

${ }^{1}$ NIHR Nottingham Hearing Biomedical Research Unit, Division of Clinical Neuroscience, School of Medicine, University of Nottingham, Nottingham, UK

${ }^{2}$ Nottingham University Hospitals NHS Trust, Nottingham, UK

${ }^{3}$ School of Health Sciences, University of Nottingham, Nottingham, UK

Corresponding author:

Melanie Ferguson

NIHR Nottingham Hearing Biomedical Research Unit

113 The Ropewalk

Nottingham, UK

NG1 5DU

Tel: $(+44) 1158232619$

Fax: $(+44) 1158232615$

Email: Melanie.Ferguson@nottingham.ac.uk

Running head:

Internet competency in hearing aid users

Key words:

Hearing aids

Hearing loss

Practical hearing aid knowledge

Hearing aid handling skills

Self-efficacy 
Internet competency in hearing aid users

\title{
Purpose
}

To assess whether internet competency predicts practical hearing aid knowledge and handling skills in first-time hearing aid users.

\begin{abstract}
Method
The design was a prospective, randomized controlled trial of a multimedia educational intervention consisting of interactive video tutorials (or reusable learning objects: RLOs). RLOs were delivered through DVD for TV or PC, and online. Internet competency was measured at the hearing aid fitting appointment, whereas hearing aid knowledge and practical handling skills were assessed six-weeks post-fitting.
\end{abstract}

\begin{abstract}
Results
Internet competency predicted practical hearing aid knowledge and handling skills, controlling for age, hearing sensitivity, educational status and gender, for the group that received the RLOs. Internet competency was inversely related to the number of times the RLOs were watched.
\end{abstract}

\section{Conclusion}

Associations between internet competency and practical hearing aid knowledge, handling skills, and watching the RLOs fewer times may have arisen because of improved selfefficacy. Therefore, first-time hearing aid users that are more competent internet users may be better equipped to apply newly learnt information to effectively manage their hearing loss. 
Internet competency in hearing aid users

\section{Introduction}

An educational program for first-time hearing aid users has been previously developed that included practical and psychosocial aspects of hearing aids and communication (Ferguson et al., 2015). The program (branded as C2Hear) is based on the concept of reusable learning objects (RLOs), which are 'chunks' of interactive multimedia learning, containing highly visual components (e.g. animations, video clips, patient testimonials), and based on pedagogical principles (Windle et al., 2010). Previous research in 2010/11 had shown that PC and internet use was low in the typical first-time hearing aid user age-group (70-74 years) in the UK, with $36.3 \%$ and $17.5 \%$ reporting that they used a PC or the Internet respectively (Henshaw et al., 2012). Consequently, the RLOs were developed for delivery through DVD for TV or PC, and via the internet, to maximize accessibility by first-time hearing aid users. This required the RLOs to be developed for a DVD platform, which inherently limited interactivity and individualization (Ferguson et al., 2016a).

Following development of the RLOs, a randomized control trial (RCT) was conducted to evaluate their effectiveness. Half (50.6\%) of the participants chose the DVD for TV mode of delivery, $15.2 \%$ opted for delivery via DVD for PC and $32.9 \%$ chose to view them online. The RCT showed a number of benefits for first-time hearing aid users who received the RLOs (RLO+), in comparison to the standard management condition (RLO-). Six weeks post-fitting, the RLO+ intervention group had significantly greater knowledge on how to use their hearing aids and were more confident and skilled at using their hearing aids (Ferguson et al., 2015; Ferguson et al., 2016a). Self-reported hearing aid use was also significantly greater in the RLO+ group, but only for 'suboptimal' users. 
Internet competency in hearing aid users

Since the RLOs were originally developed in 2011/12, the UK has seen a year-on-year increase in internet use in 55-74 year olds $(2010=61 \% ; 2012=70 \% ; 2014=78 \%)$ (United Economic Commission for Europe, 2015). Similarly, there has been a dramatic increase in computer use in $65+$ year olds, from $9 \%$ (2006) to $45 \%$ (2015), while in 55-64 year olds use has increased from 36\% (2006) to 72\% (2015) (UK Office for National Statistics, 2015). The increasing digital competency in older adults suggests the time is right to focus on the development of internet-delivered hearing-related interventions (Ferguson \& Henshaw, 2015). Indeed, there are now a number of online rehabilitation programs that have been developed for adult hearing aid users (Thorén et al., 2014) and people with tinnitus (Greenwell et al., 2015).

It is likely that a number of factors, such as age and internet competency, impact use and acceptance of internet-based hearing interventions (Moore et al., 2015). There is also some value in understanding whether internet competency affects the effectiveness of digital interventions, as it may serve as a potential barrier to an older population. Furthermore, an improved understanding of the impact of internet competency will inform how interventions should be developed and optimally delivered to people with hearing loss. Thus, the aim of this study was to assess whether internet competency was predictive of practical hearing aid knowledge and handling skills following intervention of the multimedia educational RLOs.

\section{Method}

We report unpublished data from the original study (Ferguson et al., 2016a), evaluating the RLOs in first-time hearing aid users. The design was a single-center, prospective clinically registered RCT (http:/www.isrctn.com/ISRCTN11486888) of 203 first-time hearing aid users (mean age $=67.8$ years, $\mathrm{SD}=9.5$, range $=42.2-94.8$; mean better ear average $\mathrm{e}_{0.25-4 \mathrm{kHz}}=32.0$ 
Internet competency in hearing aid users

$\mathrm{dB}$ HL, $\mathrm{SD}=8.7$, range=6-74) with two arms: (i) the intervention group received the RLOs immediately following their hearing aid fitting appointment (RLO+, $\mathrm{n}=103$ ); (ii) the control group received standard clinical management only (RLO-, $n=100)$. A detailed account of the study methods is reported in Ferguson et al. (2016a).

Internet competency was rated by participants at the hearing aid fitting appointment on a validated three-category scale (Never used, Beginner, or Competent; Henshaw et al., 2012 . Educational status was also reported on a three-category scale (Secondary school, up to 15 years; A-Level/Diploma or equivalent; Degree level or above - corresponding to junior high, senior high, college/university respectively). Outcomes were assessed six-weeks post-hearing aid fitting, and included self-report questionnaires on knowledge of practical and psychosocial aspects of hearing aids and communication (Hearing Aid and Communication Knowledge questionnaire, HACK; Ferguson et al., 2015), and practical hearing aid handling skills (Practical Hearing Aid Skills Test, PHAST; Desjardins \& Doherty, 2009). All outcome measures were completed by two audiologists at the Nottingham Hearing Biomedical Research Unit, who were blind to the participant's group allocation at the beginning of the session.

\section{Statistical Analysis}

A difference in internet competency within each group was tested using the Chi-square, and between RLO+ and RLO- groups using an independent samples Mann-Whitey U test.

Spearman's rho correlation coefficients were used to test associations between internet competency and demographic characteristics (across the entire sample) and RLO mode of delivery (RLO+ only), in addition to hearing aid knowledge and skills (separately for RLO+/groups). For each questionnaire, $p$-values were Holm-Bonferroni corrected for each sub-scale 


\section{American Journal of Audiology}

Internet competency in hearing aid users

(Aickin \& Gensler, 1996; Holm, 1979). For all significant correlations ( $p \leq .05$ ), multiple linear regression analysis tested whether internet competency predicted outcomes six-weeks post-hearing aid fitting.

\section{Results}

A significantly lower proportion of individuals reported that they had either 'never used' the internet (RLO+, 20.1\%; RLO-, 22.1\%) or were beginners (RLO+, 28.7\%; RLO-, 31.6\%), in comparison to those reporting that they were 'competent' users (RLO+, 51.1\%; RLO-, $46.3 \%)$ in both the RLO $+\left(X^{2}(2, N=94)=14.32, p=.001\right)$ and RLO- groups $\left(X^{2}(2\right.$, $N=95)=8.48, p=.014$ ) (Table 1). Internet competency did not significantly differ between the RLO+/- groups $(U=4254.5, p=.543)$.

Across the whole sample, greater internet competency was significantly correlated with a younger age $\left(R_{s}(189)=-.29, p<.001\right)$, better-hearing threshold $\left(R_{s}(189)=-.23, p=.001\right)$, higher educational status $\left(R_{s}(155)=.23, p=.004\right)$, and with being male $\left(R_{s}(189)=.17, p=.021\right)$. In the $\mathrm{RLO}+$ group, greater internet competency was also strongly associated with selecting the internet mode of delivery $\left(R_{s}(99)=.62, p<.001\right)$.

For the RLO+ group, greater internet competency was associated with significantly greater knowledge of practical hearing aid issues $\left(R_{s}(56)=.34, p=.010\right)$ (Fig. 1A), but not psychosocial issues $\left(R_{s}(56)=.17, p=.190\right)$. Greater internet competency was also associated with better practical hearing aid handling skills at follow-up $\left(R_{s}(74)=.27, p=.02\right)$ (Fig. 1B). In contrast, greater internet competency was significantly associated with watching the RLOs fewer times $\left(R_{s}(66)=-.33, p=.006\right)$ (Fig 1C). Internet competency was the only significant predictor of practical hearing aid knowledge $(\beta=5.47, t(54)=2.76, p=.008)$, accounting for 
Internet competency in hearing aid users

$12 \%$ of the variance. Internet competency also significantly predicted practical hearing aid handling skills $\left(\beta=3.0, t(72)=2.6, p=.011, R^{2}=.09\right)$, in addition to times watched $(\beta=-3.39$, $\left.t(65)=-2.95, p=.004, R^{2}=.12\right)$. No additional variance was accounted for by age, hearing threshold, level of education or gender when entered into each model $(p \geq .080)$.

There was no significant relationship between internet competency and practical hearing aid knowledge and handling skills ( $p \geq .11)$ in the RLO- group.

\section{Discussion}

In this study we assessed the extent to which self-reported internet competency predicted practical hearing aid knowledge and handling skills. Greater internet competency predicted superior practical hearing aid knowledge and handling skills. Internet competency was also predictive of how many times the RLOs were watched, with greater competency associated with watching the RLOs fewer times. Furthermore, internet competency was a significant predictor of hearing aid knowledge and skills after controlling for demographic characteristics (age, hearing threshold, educational status, gender), which have all been shown previously to be related to internet use (Henshaw et al., 2012). However, greater internet competency predicted superior practical hearing aid knowledge and handling skills only in individuals that received the RLO intervention (RLO+). While internet competency did not differ significantly between RLO+/- groups, no relationship between internet competency and outcomes was found for the standard clinical management control group (RLO-).

One potential explanation for why greater internet competency predicted hearing aid handling skills and knowledge in the RLO+ group only may be attributed to perceived self-efficacy (or 


\section{American Journal of Audiology}

Internet competency in hearing aid users

confidence) to perform a specific behavior. In the audiological field, the impact of selfefficacy on health behavior and patient outcomes has been increasingly recognized (Coulson et al., 2016), with recent research showing that individuals with higher levels of self-efficacy for using hearing aids are more likely to obtain them and become successful users (Ferguson et al., 2016b; Ferguson et al., in press; Hickson et al., 2014; Meyer et al., 2014). The use of digital technologies in the treatment and management of hearing loss has also been shown to improve patient compliance to hearing healthcare treatment, which has been attributed to increased self-efficacy (Amlani, 2015).

Although self-efficacy for hearing aids was not specifically tested in this study, our results may provide additional support for the argument that individuals in the RLO+ group that had greater internet competency may also have had greater self-efficacy to use the intervention, and subsequently perform hearing aid related behaviors (e.g. changing the battery, using the telephone, etc.). This may have later manifested itself in terms of superior practical hearing aid knowledge and handling skills. This implies that the provision of the RLO intervention not only improves hearing aid handling skills and knowledge relative to standard care (Ferguson et al., 2015), but is enhanced further with increasing internet competency. Differences between intervention groups may have arisen as a consequence of: (i) additional information being covered in the RLOs that was not given by the audiologist during the fitting appointment due to limited time constraints (Ferguson et al., 2015); (ii) the RLOs providing additional cues that improved retention of the information provided; (iii) the RLOs facilitating more realistic expectations that resulted in better outcomes (Ferguson et al., in press); or (iv) a combination of (i), (ii) and/or (iii). 
Internet competency in hearing aid users

Greater self-efficacy may also help to explain why the RLOs were watched fewer times in people with greater internet competency, because they potentially had greater confidence in their ability to use the RLOs. Greater computer literacy has been shown to be associated with greater computer self-efficacy, which impacts user acceptance of web-based interventions (Moore et al., 2015). Therefore, it is likely that individuals with greater internet competency may have familiarized themselves more readily with the content of the RLOs, resulting in fewer times watched. As a consequence, considerations should be made when developing digital interventions to ensure that they are both accessible and engaging for users with differing levels of competency, such as through the delivery of mobile-enabled RLOs (or mRLOs) that can be used via smartphones, tablet computers, and PCs.

\title{
Future directions
}

The evidence presented here, in addition to research supporting the effectiveness of the RLOs (Ferguson et al., 2016a) and that from other Interactive Health Communication Applications (see systematic review, Murray, Burns, See, Lai, \& Nazareth, 2005), suggests that the use of digital interventions will provide additional benefits to hearing aid users, which will likely to extend to their family members and friends. Furthermore, it is unlikely that digital skills will pose a significant barrier in the self-management of age-related hearing loss in the future, given that computer and internet literacy skills are on the rise in 55+ year olds (UK Office for National Statistics, 2015; United Economic Commission for Europe, 2015).

\author{
With this in mind, based on participant feedback, the RLOs have been branded and \\ distributed commercially as $\mathrm{C} 2 \mathrm{Hear}$, and are freely available via the internet, \\ (https://www.youtube.com/, search C2HearOnline). We are also currently developing a \\ platform that will deliver m-RLOs. The content is initially being designed for communication
}




\section{American Journal of Audiology}

Internet competency in hearing aid users

partners of hearing aid users in the form of a web-based app, with three RLOs (Hearing loss and its consequences; Communication tactics; Psychosocial aspects of hearing loss). The app will be designed so that it can be presented on multiple mobile devices and computer browsers, providing the potential to download self-contained RLOs. We anticipate that this mode of delivery also has greater potential to enhance interactivity and accessibility for endusers. This app will form the foundation for further developments aimed at hearing aid users, where we plan to develop an m-RLO resource that can be individually tailored to meet the needs of the end-user. It is also expected that the use of internet-based interventions under development will enable individualized learning and recall of relevant information in situations where it is needed 'on the go', either within or outside the home environment.

To summarize, while first-time hearing aid users with greater internet competency who received the RLO intervention had better practical hearing aid knowledge and handling skills, they also watched the RLOs fewer times. We suggest that these findings reflect the possibility that first-time hearing aid users who are more competent internet users are better equipped to apply newly learnt information to effectively manage their hearing loss. Nevertheless, given that digital literacy skills continue to increase year-on-year in older adults, the time is now right to design and deliver internet-based interventions in this population. Internet-based interventions may also have the capacity to provide additional benefits, not only with regards to self-management of hearing loss and hearing aids, but will likely provide a means of personalizing healthcare delivery to further enhance hearing outcomes. 
Internet competency in hearing aid users

\section{Acknowledgements}

Part of this work was presented at the Second International Meeting on Internet \& Audiology, Eriksholm Research Centre, Oticon A/S, Snekkersten, Denmark, September 24-25, 2015.

This paper presents independent research funded by the National Institute for Health Research (NIHR) under its Research for Patient Benefit (RfPB) Program (Grant Reference Number PB-PG-0909-20294). The views expressed are those of the authors and not necessarily those of the NHS, the NIHR or the UK Department of Health. We thank the research team members Marian Brandreth, Holly Thomas, Paul Leighton, James Henderson and Michael Taylor. In addition, we gratefully acknowledge the audiologists at Nottingham Audiology Services who recruited the patients, as well as our patient and public involvement panel (Anne Darby, Tina Wales and Rachel Ravenlock).

\section{Conflicts of interest:}

The Nottingham University Hospitals NHS Trust and University of Nottingham will receive a proportion of any royalties from the sale of the C2Hear RLOs (DVD format). 
Internet competency in hearing aid users

\section{Figure Legend}

Figure 1. Mean scores (\%) for A. practical hearing aid knowledge, and B. hearing aid handling skills; C. Mean total number all RLOs watched, for each internet competency category rated by participants in the RLO+ group. $\mathrm{HACK}=$ Hearing Aid and Communication Knowledge questionnaire; PHAST = Practical Hearing Aid Skills Test. 
Internet competency in hearing aid users

\section{References}

Aickin, M., \& Gensler, H. (1996). Adjusting for multiple testing when reporting research results: The Bonferroni vs Holm methods. American Journal of Public Health, 86(5), 726-728.

Amlani, A. M. (2015). Improving Patient Compliance to Hearing Healthcare Services and Treatment through Self-Efficacy and Smartphone Applications. The Hearing Review, $22(2), 16-20$.

Coulson, N., Ferguson, M. A., Henshaw, H., \& Heffernan, E. (2016). Applying theories of health behaviour and change to hearing health research: Time for a new approach. International Journal of Audiology, advance online publication. DOI: 10.3109/14992027.14992016.11161851.

Desjardins, J. L., \& Doherty, K. A. (2009). Do experienced hearing aid users know how to use their hearing aids correctly? American Journal of Audiology, 18, 69-76.

Ferguson, M. A., Brandreth, M., Brassington, W., \& Wharrad, H. (2015). Information retention and overload in first-time hearing aid users: An interactive multimedia educational solution. American Journal of Audiology, 24, 329-332.

Ferguson, M. A., Brandreth, M., Leighton, P., Brassington, W., \& Wharrad, H. (2016a). A randomized controlled trial to evaluate the benefits of a multimedia educational programme for first-time hearing aid users. Ear and Hearing, 37(2), 123-136.

Ferguson, M. A., \& Henshaw, H. (2015). Computer and internet interventions to optimise listening and learning for people with hearing loss: Accessibility, use and adherence. American Journal of Audiology, 24, 338-343.

Ferguson, M. A., Maidment, D. W., Russell, N., Gregory, M., \& Nicholson, N. R. (2016b). Motivational engagement in first-time hearing aid users: A feasibility study. 
Internet competency in hearing aid users

International Journal of Audiology, advance online publication.

DOI:10.3109/14992027.14992015.11133935.

Ferguson, M. A., Woolley, A., \& Munro, K. J. (in press). The impact of self-efficacy, expectations and readiness on hearing aid outcomes. International Journal of Audiology.

Greenwell, K., Featherstone, D., \& Hoare, D. J. (2015). The Application of intervention coding methodology to describe the tinnitus e-programme, an internet-delivered selfhelp intervention for tinnitus. American Journal of Audiology, 24(3), 311-315.

Henshaw, H., Clark, D., Kang, S., \& Ferguson, M. (2012). Computer Skills and Internet Use in Adults Aged 50-74 Years: Influence of Hearing Difficulties. Journal of Medical Internet Research, 4(4), e113,111-114.

Hickson, L., Meyer, C., Lovelock, K., Lampert, M., \& Khan, A. (2014). Factors associated with success with hearing aids in older adults. International Journal of Audiology, 53(1), S18-S27.

Holm, S. (1979). A simple sequentially rejective multiple test procedure. Scandinavian Journal of Statistics, 6(2), 65-70.

Meyer, C., Hickson, L., \& Fletcher, A. (2014). Identifying the barriers and facilitators to optimal hearing aid self-efficacy. International Journal of Audiology, 53(1), S28-S37.

Moore, A. N., Rothpletz, A. M., \& Preminger, J. E. (2015). The Effect of Chronological Age on the Acceptance of Internet-Based Hearing Health Care. American Journal of Audiology, 24(3), 280-283.

Murray, E., Burns, J., See, T. S., Lai, R., \& Nazareth, I. (2005). Interactive Health Communication Applications for people with chronic disease. Cochrane Database of Systematic Reviews, 19(4). 
Internet competency in hearing aid users

Thorén, E. S., Öberg, M., Wänström, G., Andersson, G., \& Lunner, T. (2014). A randomized controlled trial evaluating the effects of online rehabilitative intervention for adult hearing-aid users. International Journal of Audiology, 53(7), 452-461.

UK Office for National Statistics. (2015). Internet Access - Households and Individuals 2015. Retrieved from United Kingdom: http://www.ons.gov.uk/ons/rel/rdit2/internetaccess---households-and-individuals/2015/stb-ia-2015.html

United Economic Commission for Europe. (2015). Statistical database: Percentage of population using Internet by age, sex, variable, county and year. Retrieved from http://w3.unece.org/PXWeb2015/pxweb/en/STAT/STAT_30-GE_ 09Science_ICT/02_en_GEICT_InternetUse_r.px/?rxid=9d2304d4-bc92-41ce-b0e9$\underline{38827 \mathrm{cfdf} 89 \mathrm{e}}$

Windle, R. J., McCormick, D., Dandrea, J., \& Wharrad, H. (2010). The characteristics of reusable learning objects that enhance learning: A case-study in health-science education. British Journal of Educational Technology, 42(5), 811-823. 

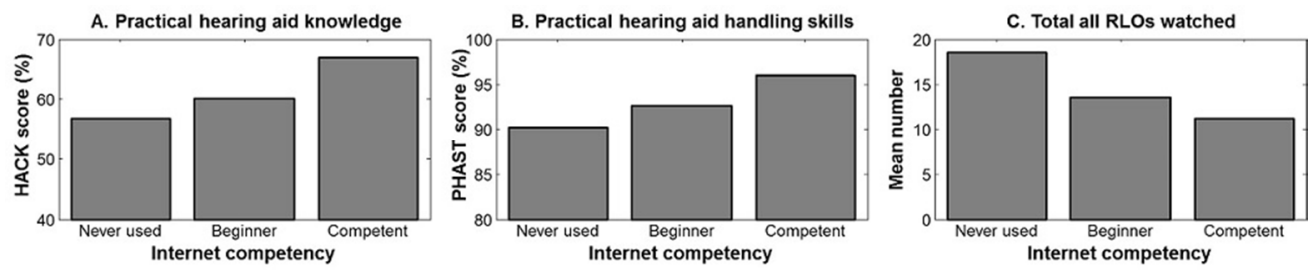

1

Fig.

264×55mm (96 x 96 DPI) 